\title{
ON FUNCTIONAL EQUATIONS RELATED TO MIELNIK'S PROBABILITY SPACES
}

\author{
C. F. BLAKEMORE AND C. V. STANOJEVIC
}

ABSTRACT. It is shown that the method used by C. V. Stanojevic to obtain a characterization of inner product spaces in terms of a Mielnik probability space of dimension 2 does not admit a generalization to dimension $n>2$.

Let $f:[0,2] \rightarrow[0,1]$ be continuous and strictly increasing with $f(0)$ $=0$ and $f(2)=1$. The class of all such functions $f$ will be denoted by $F$. Likewise, let $g:[0,2] \rightarrow[0,2]$ be continuous but strictly decreasing with $g(0)=2$ and $g(2)=0$. Similarly, the class of all such functions $g$ will be denoted by $G$. In [1] it is proved that the functional equation

$$
f+f \circ g=1
$$

where $(f \circ g)(t)=f[g(t)]$ has a solution $f \in F$ if and only if $g \in G$ is an involution, i.e., $g \circ g=e$ where $e$ is the identity function on $[0,2]$. Using this result it is also shown that a normed real linear space $N$ is an inner product space if and only if for some $f \in F,(S, f(|x+y|))$ is a Mielnik probability space [2] of dimension 2 . The functional equation $(*)$ served as a tool to obtain a new characterization of inner product spaces. In this note we consider the possibility of extending this characterization of inner product spaces to the case where $p$ is a probability function generated by an appropriate function $f$ and $(S, p)$ is of dimension $>2$.

Let $g^{(m)}$ denote $m$ iterations of a function $g: I \rightarrow I$ where $I$ is some interval. Also, suppose $g^{(n)}=e$ where $e$ is the identity function on $I$ and $n$ is some positive integer. We shall show that the generalized functional equation

$$
f+f \circ g+f \circ g^{(2)}+\cdots+f \circ g^{(n-1)}=1
$$

(where $f$ and $g$ are functions belonging to a suitable generalization of the classes $F$ and $G$ defined earlier) collapses. In other words, the method from [1] cannot be extended in a straightforward manner to the case when $(S, p)$ is of dimension $>2$. The following theorem (for a similar result for homeomorphisms see [3]) is the key to our result:

Received by the editors July 16, 1974. $46 \mathrm{C} 10$.

AMS (MOS) subject classifications (1970). Primary 39A15, 26A18; Secondary

Key words and phrases. Functional equations, Mielnik probability spaces. 
Theorem. Let $h: I \rightarrow I$ be a function where $I$ is an interval. If $h$ is continuous and if for some $m \geq 2, h^{(m)}=e$, then $h$ is an involution, i.e., $h \circ h=e$.

Proof. Let $h(x)=h(y)$. Then, since $h^{(m)}=e$, we have $h^{(m)}(x)=$ $h^{(m)}(y)$ implies $x=y$ and thus $h$ is one-to-one. Hence, since $h$ is continuous, $h$ is strictly monotone. First we consider the case where $h$ is strictly increasing. Then from $h(x)>x$ it follows that $x=h^{(m)}(x)>$ $h^{(m-1)}(x)>\ldots>h(x)>x$ which is a contradiction. The contradiction also follows from the assumption $h(x)<x$. Hence $h(x)=x$ for all $x$ in $I$ and $h \circ h=e$. Next we consider the case where $h$ is strictly decreasing. If $x$ $<y$, then $h(x)>h(y)$ and $h^{(2)}(x)<h^{(2)}(y)$. Hence $b^{(2)}$ is strictly increasing. But $\left(h^{(2)}\right)^{(m)}=\left(h^{(m)}\right)^{(2)}=e^{(2)}=e$. Applying the first case to $h^{(2)}$ we get $h^{(2)}=e$. Therefore $h^{(2)}=e$ and $h$ is an involution.

In particular, our theorem shows that the function $g: I \rightarrow I$ appearing in our generalized functional equation $(* *)$ must be an involution. Thus $(* *)$ becomes $n(f+f \circ g) / 2=1$ for $n$ even and $(n+1) f / 2+((n-1) / 2) f \circ g=1$ for $n$ odd. Now if we want to extend the result from [1] to the $n$-dimensional case we have to have $(* *)$ since it is equivalent to Axiom (C) of Mielnik [2]. This shows that there is not a trivial extension to dimension $n$ using the procedure from [1].

\section{REFERENCES}

1. C. V. Stanojevic, Mielnik's probability spaces and characterization of inner product spaces, Trans. Amer. Math. Soc. 183 (1973), 441-448.

2. B. Mielnik, Geometry of quantum states, Comm. Math. Phys. 9 (1968), 5580. MR $37 \# 7156$.

3. N. McShane, On the periodicity of homeomorphisms of the real line, Amer. Math. Monthly 68 (1961), 562-563. MR 24 \#A199.

DEPARTMENT OF MATHEMATICS, UNIVERSITY OF NEW ORLEANS, NEW ORLEANS, LOUISIANA 70122

DEPARTMENT OF MATHEMATICS, UNIVERSITY OF MISSOURI AT ROLLA, ROLLA, MISSOURI 65401 